\title{
Adaptive hybrid technique for face recognition
}

\author{
Hazeem B. Taher ${ }^{1,}$ Kadhim M. Hashim ${ }^{2}$, Atheer Yousif Oudah ${ }^{3}$ \\ ${ }^{1,2,3}$ Computer Science Department, College of Education for pure Sciences, Thiqar University, Iraq
}

\section{Article Info}

Received Jan $14^{\text {th }}, 2019$

\section{Keyword:}

Pattern recognition

Face Recognition

Singular Value

Decomposition (SVD)

Standard Deviation (SD)

\begin{abstract}
One of the most important biometric features for personal identification is the face. In current paper, a new method of face verification upon on singular value decomposition (SVD) and standard deviation (SD) would be described. Due to many variations in real-life such as pose, illumination, or facial expression, there would be difficulty of face recognition. It should be mentioned that there are many approaches for face recognition, however, there is no one could be considered as the most suitable for many situations. One of the methods used is Singular value vector for an image detecting, but the drawback of this approach is the low rate of recognition, where one scale singular value vector is used for face acknowledgment. There an algorithm has been developed to expand the rate of the recognition. In this paper, an approach has been proposed to associate two feature sets obtained from SVD and SD method. It has noticed a good recognition rate could be obtained from the experimental results, where approximately more that $97.5 \%$ recognition rate has obtained on the ORL data base. The results from current proposed method have matched with some techniques and it has shown that this method is better than the existing approaches. An extensive experiment has demonstrated not only better performance, but it offers a great likely to achieve equivalent performance to other categories of state-of-the-art methods.
\end{abstract}

\section{Corresponding Author:}

Hazeem B. Taher ${ }^{1}$

Computer Science Department,

College of Education for pure Sciences,

Thiqar University, Iraq

Email: hazeem.sci@utq.edu.iq

\section{Introduction}

The face recognition method which been studied here is considered as one of the most authentic biometric approaches. It is a powerful application of Pattern recognition and Image analysis. Verification and identification are the two main tasks of the face recognition. Where the face verification aimed to match exactly a face of images with the template face of images whose character being claimed. While, face identification objective is that there is $1: \mathrm{N}$ problems and this would compare a query face image against all image templates in a face database. Because of the wide range of marketable and edict enforcement applications such as actual scientific identification, entrance of control, border of surveillance and human communications, the machine recognition became crucial tool. Basically, there are three approaches of face recognition, which are holistic methods and this method would be essentially used whole face area as required input to what has been knowing as a recognition system. Second method is Feature-based, this method uses the feature of the face such a nose, eyes and mouth. The third method is Hybrid method which uses both face feature and whole face area to recognize the face [2]. Since of founding many images variation in real-life, such as lighting, brightness, facial appearance, as well as, partial obstruction and imprecise local that express in many different of face area. face recognition is considered as a difficult problem [3]. Many systems for 
face recognition have been projected in the literatures [2]. The principal component analysis (PCA) considered as the most popular approach, which is holistic based method and it is suggested by [4]. The objective of this method it to transform 2D face pictures for 1D vector. Obtained vector would have highlydimensional space where assessment of covariance matrix will became more difficult due to giant size or inordinate numbers of training samples. Furthermore, there has to compute the eigenvectors of a huge covariance of matrix, and this time consuming process. Linear discriminant of analysis (LDA) is well documented and used as traditional technique. It is used for feature abstraction and dimension dropping and utilized for a while to organize the problems [5]. Algebraic features (AF) are effective feature in objective classification for example, doing to achieve some of face recognition, and that was suggested by Hong [6]. He suggested a utilizing what is known as singular value decomposition (SVD) as basic for recognition method, and this is based on remarkable values of feature vectors. Effective of SVD process has tested in [6] and [7]. Fusion of some selected left and/or right singular vectors of SVD and DCT-RLDA can generate superior results on face images as compared to their individual counterpart [8]. In this paper, an approach has been developed to mix two sets of features that are obtained from SVD and SD method. The exactness of projected recognition approaches is compered to exist techniques. The organization of the paper is as follows, section 2 describe the Singular Value Decompositions. Then the Standard Deviation has introduced in section 3. In Section 4 Dissimilarity measurement is described. Section 5 describes the proposed approach. The main results of current experiment are presented in section 6 and conclusions which is drawn are explained in section 7.

\section{Singular Value Decomposition (SVD)}

Singular Value Decompositions (SVDs) are define as an algebraic practice to factor several rectangular matrixs for the product of three other matrices. If $A \in R^{m \times n}$ is identify as a gray level facial image and $\operatorname{rank}(A)=r$, after that there are two orthogonal matrices

$$
\begin{gathered}
U=\left[u_{1}, \ldots, u_{m}\right] \in R^{m \times m}, U^{t} U=I \\
V=\left[v_{1}, \ldots, v_{n}\right] \in R^{n \times n}, V^{t} V=I
\end{gathered}
$$

and diagonal matrix

$$
\Sigma=\operatorname{diag}\left(\lambda_{1}, \lambda_{2}, \ldots, \lambda_{r}, 0, \ldots, 0\right) \in R^{m \times n}, \lambda_{1} \geq \lambda_{2} \geq \cdots \geq \lambda_{r} \geq 0
$$

such that

$$
A=U \Sigma V^{t}=\sum_{i=1}^{r} \lambda_{i} u_{i} v_{i}^{t}
$$

where $\lambda_{i}{ }^{2}$ is the eigenvalue of $A^{t} A$ in addition to $A A^{t}, \lambda_{i}$ is Singular of Value (SV) from facial pic A, $u_{i}$, $v_{i}$, are pillar of eigenvectors of $A^{t} A$ and $A A^{t}$ corresponding to eigenvalue $\lambda_{i}{ }^{2}$, respectively[9-11].

SVD has important properties and these are verified in [12]. These properties are stability, transposition invariance, rotation invariance, proportion invariance, mirror trans- form invariance. The singular values are invariant to translation, rotation, mirror transforms, and this is proven in literature [6]. It should be mention that it is stable in spite of the occurrence of other variations, such as noise or changing lighting.

\section{Standard Deviation (SD)}

Standard Deviation (SD) of our data is a measured of in what way to extent out data as in [13]. Mathematically, SDs are square root of the variances. The main character for population standard deviation is known in literature as $\sigma$. Corresponding formula for the population standard deviation $\sigma$ is:

$$
\sigma=\sqrt{\sigma^{2}}
$$

where $\sigma^{2}$ is adjustment, Variances are average of squares of the distances for each value from the mean of these values. Formula for variance is:

$$
\sigma^{2}=\frac{\sum_{\mathrm{i}=1}^{\mathrm{N}}\left(\mathrm{X}_{\mathrm{i}}-\mu\right)^{2}}{\mathrm{~N}}
$$

where $X_{i}$ individual values, $\mu$ mean of the data set. $N$ is number of elements in the data set $[13,14]$. 


\section{Dissimilarity measurement}

In our study we are interested to city of block distance measurement, in our metric, the distances between 2 vectors are summations of common absolute alterations of their essentials. Mathematically, distances between 2 vectors $\mathrm{u}=\left(\mathrm{u}_{1}, \mathrm{u}_{2}, \ldots, \mathrm{u}_{\mathrm{N}}\right)$ and $\mathrm{v}=\left(\mathrm{v}_{1}, \mathrm{v}_{2}, \ldots, \mathrm{u}_{\mathrm{N}}\right)$ which are given by [15]:

$$
\mathrm{D}(\mathrm{u}, \mathrm{v})=\sum_{\mathrm{i}=1}^{\mathrm{N}}\left|\mathrm{u}_{\mathrm{i}}-\mathrm{v}_{\mathrm{i}}\right|(4)
$$

\section{The Proposed Approach}

Figure (1) shows the block diagram of the proposed approach. It consists of the following steps:

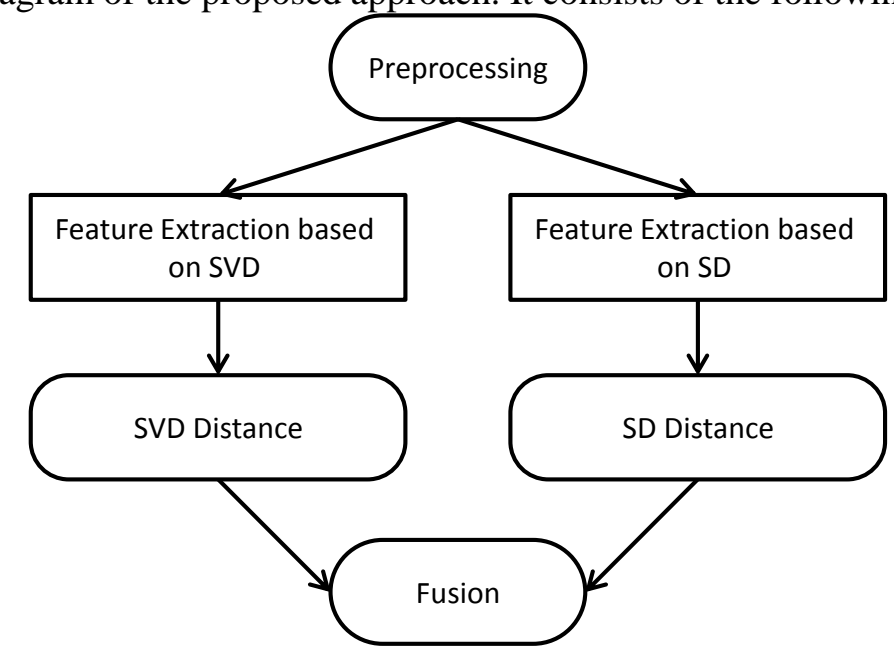

Figure 1. Block diagram of the proposed approach

1-Preprocessing: First, the image from the database is obtained and improved to gray scale. Then the picture is divided into (4x4) 16 blocks with (28x23) pixels (See Figure (2)).

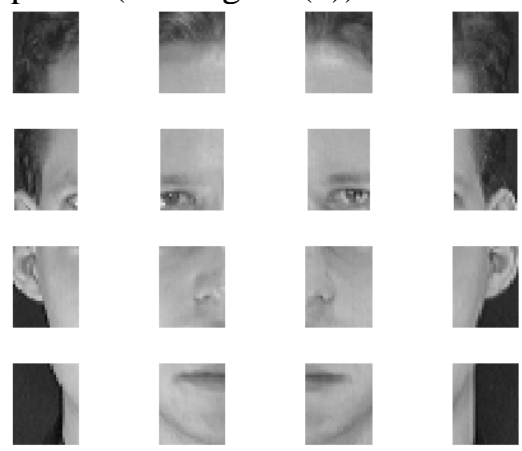

Figure 2. Example the face image is divided

2-Feature Extraction based on SVD: SVD for each block obtained in the preprocessing step is calculated. Then we used the singular values as the feature vectors.

3-Feature Extraction based on SD: SD values for each column of each block obtained in the preprocessing step is calculated and use them as the feature vectors.

4-Computation of Dissimilarity Measure: In SVD-Based Features Extraction, we have a vector V that contain features of consistent to examined face and a matrix $F_{k}(k=1,2, \ldots, M)$, such as $M$ represents numbers of training faces. The judgment between $\mathrm{F}_{\mathrm{k}}$ and $\mathrm{v}$ is done by computing the distances amongst them to obtain the distance vector $D_{S V D}=\left(d_{1}, d_{2}, \ldots, d_{M}\right)$. $D_{S V D}$ would be characterized the score that be collected with SD. In SD-base Features Extraction, now comparison between $F_{k}$ and $v$ is done by manipulative the distances between them to obtain the distance vector $D_{S D}=\left(d_{1}, d_{2}, \ldots, d_{M}\right)$ that characterized the score that be combined to SVD. 
5- Fusion Method: After computing 2 distances vectors, we collected the two main results vectors $\mathrm{D}_{\mathrm{SVD}}$ and $\mathrm{D}_{\mathrm{SD}}$ in order to calculate combined vector $\mathrm{D}_{\text {Fusion. }}$. Finally, $\mathrm{D}_{\text {Fusion }}$ are calculated as multiplication of 2 distances vectors. We selected the conforming class according to the smallest value of $\mathrm{D}_{\text {Fusion. }}$.

$$
D_{\text {Fusion }}=\left(\left(D_{1}^{S V D} \cdot D_{1}^{S D}\right),\left(D_{2}^{S V D} \cdot D_{2}^{S D}\right), \ldots,\left(D_{M}^{S V D} \cdot D_{M}^{S D}\right)\right)(5)
$$

\section{Experiment results}

The standard database Olivetti Research Laboratory (ORL) [16] is selected to test recognition accuracy of the new proposed approach. In the ORL database, it has been approved that there are 10 dissimilar images for each of the 40 different themes. Differences in face expression are present (smile and/or non-smile face as well as open and/or close eyes), and face specifics such as wearing glasses and/ or no glasses on. Most of these pictures were taken against black standardized background with the subjects in frontal position and an up-right, all of these images are tolerance for some tilting's and revolutions of up to about $20^{\circ}$. Figure (3) is a picture of a person shown in the database. Database are component from 400 face images. The resolution of all images is $112 \times 92$, and 8 -bit grey levels.
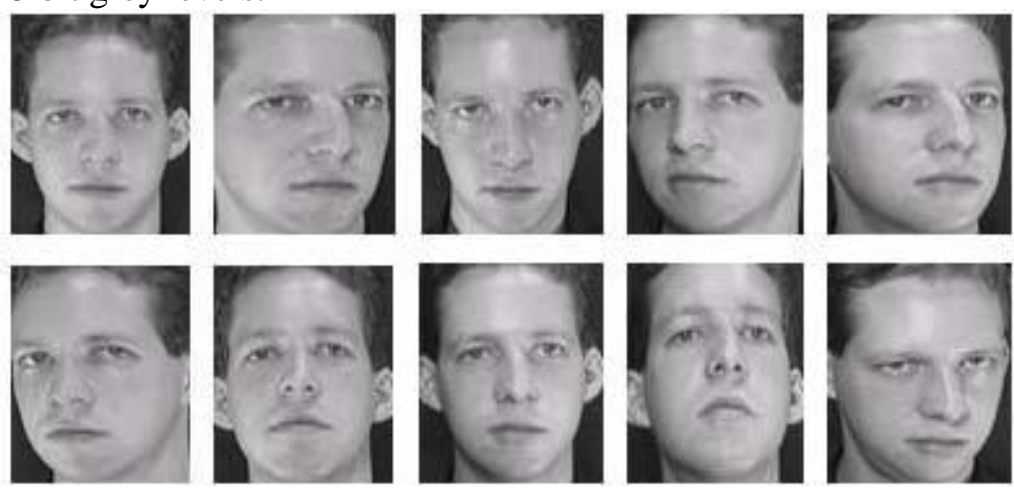

Figure 3. Images of one person in the ORL database

In our experiments, 5 images from each person were chosen as training group and the rest are the testing images. The entire number of training and testing images in the current study is 200.Table (1) shows the recognition rate of SVD, SD and proposed approach for randomly six training sets. As Table (1) show, the recognition rate of the proposed approach is better than the SVD and SD. The recognition rate of suggested approach is $2.5 \%$ higher than SVD and $8 \%$ than SD.

Table 1. Recognition rates of SVD, SD and proposed approach

\begin{tabular}{|c|c|c|c|}
\hline Filenames of training set & SVD & SD & Proposed Approach \\
\hline a1, a2, a3, a, and a5 & $95 \%$ & $89.5 \%$ & $97.5 \%$ \\
\hline a1, a2, a3, a9, and a10 & $93.5 \%$ & $90 \%$ & $98 \%$ \\
\hline a1, a2, a, a7, and a9 & $96.5 \%$ & $92 \%$ & $97.5 \%$ \\
\hline a2, a, a5, a6, and a10 & $97 \%$ & $92.5 \%$ & $98.5 \%$ \\
\hline a1, a3, a, a5, and a8 & $97 \%$ & $91.5 \%$ & $97.5 \%$ \\
\hline a2, a5, a6, a7, and a8 & $95 \%$ & $93 \%$ & $96.5 \%$ \\
\hline
\end{tabular}

The presentation of the anticipated method is analyzed against particular of the available methods. Table (2) shows the recognition accuracy of some well-known face recognition methods on ORL database wherein first 5 images are taken of each person in the training group and remaining all kept in the test cluster.

Table 2. Performance comparison of various face recognition approaches

\begin{tabular}{|l|c|}
\hline \multicolumn{1}{|c|}{ Method } & Recognition rate \\
\hline PCA (Eigenface) [17] & $87.8 \%$ \\
\hline LDA [18] & $91 \%$ \\
\hline SVD and DCT [19] & $91.5 \%$ \\
\hline SVD and DCT-RLDA[8] & $95 \%$ \\
\hline SVD & $95 \%$ \\
\hline
\end{tabular}




\begin{tabular}{|l|c|}
\hline SD & $89.5 \%$ \\
\hline Proposed Approach & $\mathbf{9 7 . 5 \%}$ \\
\hline
\end{tabular}

\section{Conclusion}

In this paper, SVD and SD based face recognition approaches have been proposed. The image was divided into 16 blocks and features were extracted from each block by using singular values and SD values for each column in each block. The recognition rate obtained by the proposed approaches was superior to that obtained by the individual SVD and SD method. The recognition accuracy of the recommended approach has also been thriving compared to existing methods and it is observed that on these databases of recognition accuracy for the offered method is better than accuracy of these familiar face recognition methods.

\section{References}

[1] Riddhi Patel and Shruti B. Yagnik, "A Literature Survey on Face Recognition Techniques," International Journal of Computer Trends and Technology (IJCTT), Vol. 5, No. 4, pp. 189-194, 2013.

[2] W. Zhao, R. Chellappa, P. J. Phillips and A. Rosenfeld, "Face Recognition: A Literature Survey," CM Computing Surveys, Vol. 35, No. 4, pp. 399-458, 2003.

[3] Jiazhong He and Minghui Du, "Face Recognition Based on Projection Map and SVD Method for One Training Image per Person," International Conference on Computational Intelligence for Modelling, Control and Automation, and International Conference on Intelligent Agents, Web Technologies and Internet Commerce (CIMCA-IAWTIC'05), 2005.

[4] M. Turk and A. Pentland, "Eigenfaces for Recognition," Journal of Cognitive Neurosicence, Vol. 3, No. 1, pp. 71-86, 1991.

[5] J. Lu, K. N. Plataniotis and A. N. Venetsanopoulos, "Face recognition using LDA-based algorithms," IEEE Trans., Neural Networks, vol.14, pp.195-200, 2003.

[6] Z. Hong, "Algebraic feature extraction of image for recognition," Pattern Recognition,Vol.24, pp. 211219, 1991.

[7] Y Cheng, K.Liu.J.Jang, Y.Zhang and N.Gu, "Human face recognition method based on the statistical model of small sample size," SPIE Proc: Intell. Robots and Compu. Vision,Vol.1607, pp. 85-95,1991.

[8] Messaoud B., Lamia M., Farid H., Abderrazak G. and Mohamed C. "Score Fusion of SVD and DCTRLDA for Face Recognition," In Proceedings of IEEE International Conference on Image Processing theory, Tools and Applications, p. 1-8, 2008.

[9] H. Miar-Naimi and P. Davari, "A New Fast and Efficient HMM-Based Face Recognition System Using a 7-State HMM Along With SVD Coefficients," Iranian Journal of Electrical \& Electronic Engineering, Vol. 4, Nos. 1 \& 2, 2008.

[10] Yanwei Pang, NenghaiYn, Kong Zhang, JiaweiRong and Zhengkai Liu, "Fusion Of SVD And LDA For Face Recognition," International Conference on Image Processing (ICIP), pp. 1417-1420, 2004.

[11] Jia-Zhong He, Ming-Hui Du, Sheng-Wei Pei and Quan Wan, "Face Recognition Based On Spectroface And Uniform Eigen-Space SVD For One Training Image Per Person," Proceedings of the Fourth International Conference on Machine Learning and Cybernetics, Guangzhou, pp. 18-21, 2005.

[12] M. S. Bartlell, J. R. Movellan and T. J. Se Jnow Ski, "Face Recognition by Independent Component Analysis," IEEE Transon Neural Networks, Vol. 13, No. 6, pp. 1450- 1464, 2002.

[13] Lindsay I Smith, A tutorial on Principal Components Analysis, February 26, 2002.

[14] Allan G. Bluman, Elementary Statistics A Step by Step Approach, The McGraw-Hill Companies, Inc., 2009.

[15] DK H PHM Yassin, S Hoque, and F. Deravi, "Age Sensitivity of Face Recognition Algorithms", International Conference on Emerging Security Technologies, pp. 12-15, 2013.

[16] ORL, 1992. The ORL face database at the AT\&T (Olivetti) Research Laboratory. Available from: <http://www.uk.research.att.com/facedatabase.html>.

[17] Zhi R. and Ruan Q., "Two-dimensional direct and weighted linear discriminant analysis for face recognition",Neurocomputing, pp. 3607-3611, 2008. 
[18] Zhou D., Yang X., Peng N. and Wang Y,"Improved-LDA based face recognition using both facial global and local information",Pattern Recognition Letters, pp. 536-543, 2006.

[19] Jin Zhang, You Wang, Guang Li and Walter J. Freeman, "Application of Bionic Neural Network on Face Recognition Based on SVD and DCT", World Congress on Intelligent Control and Automation, pp. 2733-2736, 2004.

[20] Hazeem B. Taher, Kadhim M. Hashem, Fatima A. Sajet, " Proposed Method For Road Detection and Following Boundaries", www.jatit.org, 2018,Vol.96, No. 18 p(6106-6116).

[21] B. Durakovic and Basic, H., "Continuous Quality Improvement in Textile Processing by Statistical Process Control Tools: A Case Study of Medium-Sized Company", Periodicals of Engineering and Natural Sciences, vol. 1, no. 1, pp. 39-47, 2013.

[22] D. Petrovic, Basic, H., Durakovic, B., and Prodanovic, S., "Science-Technology Park Ilidža as a Generator of Innovation Potential and SME's Development in Bosnia and Herzegovina", Periodicals of Engineering and Natural Sciences, vol. 1, 1 vol., no. 2, pp. 51-55, 2013.

[23] B. Durakovic, "Emerging Issues, Trends and Challenges for Sustainable Engineering", The Sixth Regional Conference on Soft Computing 2017. 2017.

[24] M. Inalpolat and Durakovic, B., "Implementation of Advanced Automated Material Handling Systems in Manufacturing Environment”, European Conference of Technology and Society - EuroTecS. 2013. 